Quantitative assessment of electrostatic embedding in Density Functional Theory calculations of biomolecular systems

J. Fattebert, R. J. Law, B. Bennion, E. Y. Lau, E. Schwegler, F. C. Lightstone

April 27, 2009

Journal of Chemical Theory and Computation 
This document was prepared as an account of work sponsored by an agency of the United States government. Neither the United States government nor Lawrence Livermore National Security, LLC, nor any of their employees makes any warranty, expressed or implied, or assumes any legal liability or responsibility for the accuracy, completeness, or usefulness of any information, apparatus, product, or process disclosed, or represents that its use would not infringe privately owned rights. Reference herein to any specific commercial product, process, or service by trade name, trademark, manufacturer, or otherwise does not necessarily constitute or imply its endorsement, recommendation, or favoring by the United States government or Lawrence Livermore National Security, LLC. The views and opinions of authors expressed herein do not necessarily state or reflect those of the United States government or Lawrence Livermore National Security, LLC, and shall not be used for advertising or product endorsement purposes. 


\title{
Quantitative assessment of electrostatic embedding in Density Functional Theory calculations of biomolecular systems
}

\author{
J.-L. Fattebert, ${ }^{* \dagger}$ R. J. Law, ${ }^{\ddagger} \S$ B. Bennion, ${ }^{\ddagger}$ E. Y. Lau, ${ }^{\ddagger}$ E. Schwegler, ${ }^{\ddagger}$ and F. C. \\ Lightstone
}

Center for Applied Scientific Computing, Lawrence Livermore National Laboratory, and Physics \& Life Sciences, Lawrence Livermore National Laboratory

E-mail: fattebert1@IInl.gov

\begin{abstract}
We evaluate the accuracy of density functional theory quantum calculations of biomolecular subsystems using a simple electrostatic embedding scheme. Our scheme is based on dividing the system of interest into a primary and secondary subsystem. A finite difference discretization of the Kohn-Sham equations is used for the primary subsystem, while its electrostatic environment is modeled with a simple one-electron potential. Force-field atomic partial charges are used to generate smeared Gaussian charge densities and to model the secondary subsystem. We illustrate the utility of this approach with calculations of truncated dipeptide chains. We analyze quantitatively the accuracy of this approach by calculating atomic forces and comparing results with full QM calculations. The impact of the choice made in terminating dangling bonds at the frontier of the QM region is also investigated.
\end{abstract}

\footnotetext{
Center for Applied Scientific Computing, Lawrence Livermore National Laboratory

${ }^{\ddagger}$ Physics \& Life Sciences, Lawrence Livermore National Laboratory

${ }^{\S}$ Current address: Evotec, Oxford, U.K.
} 


\section{Introduction}

Enzymes are the most proficient catalyst known. They are able to accelerate reactions that take as long as 78 million years to 26 milliseconds under ambient conditions corresponding to a rate enhancement of $10^{17}$. How enzymes are able to lower the reaction energy barrier is still being debated and a highly active area of study (see e.g. ${ }^{1-4}$ ). Quantum mechanical calculations of the solvated enzyme with reactant would show how the energy barrier is being lowered but even with modern computers this type of calculation is prohibitive. Most quantum mechanical studies of enzymes use a model systems of the active site, typically a few residue sidechains and reactant, in the gas-phase or with a continuum solvent (see e.g. ${ }^{5,6}$ ). Although these calculations can provide useful insights they do not take into account the heterogeneous nature of an enzyme active site which usually provides a significant contribution in lowering a reaction's activation barrier or the role dynamics can play in catalysis. ${ }^{7,8}$

Proteins are composed of amino acids linked into a linear sequence by amide bonds, also known as peptide bonds, between the amino group of one amino acid and the carboxyl group of the next amino acid. The side chains of the amino acids are diverse and can be polar or nonpolar, acidic or basic, and aromatic or aliphatic. They are capable of carrying a net electrical charge depending on the nature of the surrounding environment. These charges generate long range Coulomb potentials which affect the electronic structure tens of Angstroms away. In modeling an environment made of proteins and water, Coulomb effects will dominate long range interactions and can influence the active site of a protein.

Due to the very high computational cost of quantum calculations, modeling protein systems at the quantum mechanical level is restricted to rather small systems. While the active site, where a quantum description is required to describe the chemical reaction, is often not very large, a realistic environment surrounding that region is necessary if one hopes to attain useful information out of a small active site calculation. ${ }^{8}$ Frequently, the active site is within the whole protein such that isolating the active site for a quantum calculations (also called primary subsystem) would involve "cutting" the active site from the remaining part of the protein. It is very likely that covalent bonds 
will need to be cut. A common method to create the isolated active site is to cut the covalent bonds between the active site and the "environment" (also called the secondary subsystem) and terminate these covalent bonds in the primary subsystem with hydrogens (link atoms) to satisfy valency.

Combined quantum mechanic/molecular mechanics (QM/MM) approaches have become widely used (see e.g. ${ }^{9,10}$ for recent reviews) for modeling chemical reactions in large protein systems. They allow the simulation of a large effective system using quantum modeling for only a small subsystem, the active site, while the rest of the system is treated using a classical force field approach. Past studies using these methods were limited in the size of the QM region and utilized semi-empirical hamiltonians such as AM1 or PM3. More recently, the self-consistent-charge density functional tight-binding method is gaining popularity for biological QM/MM studies. ${ }^{11}$ However, all these methods still face serious technical difficulties in describing the interface between the QM and the MM regions when covalent bonds have to be cut, which is often the case in biochemical applications. there QM/MM methods also require coupling of two completely different models which leads to very complex simulation codes or codes coupling.

In this paper, we evaluate the accuracy of a simpler model, which avoids the whole MM forcefield bonded and Van der Waals interactions machinery, and is easy to implement in a QM code. We investigate the quantitative effect of electrostatically embedding a QM subsystem into a classical biomolecular mechanics system simulation. The QM computation for the active site is carried out in the presence of a simple one-electron potential describing the electrostatic interaction with the atoms of the secondary subsystem which accounts for the influence of the protein environment. In our approach, the partial point charges associated to the secondary subsystem in a force field model are used to build smeared Gaussian charge distributions which are then used to compute an effective electrostatic potential. Such a model obviously leads to inaccurate forces for QM atoms at the boundary of the primary subsystem where bonded interactions with MM atoms would dominate, and the coordinates of these atoms should be frozen in a molecular dynamics simulation. We focus however on the accuracy of forces deeper inside the QM region, at the active site of interest. A similar study was carried out by Solt et al. ${ }^{12}$ in a full QM/MM framework where they 
attempt to assess in a systematic manner simulation errors at the center of the QM region as a function of the size of that region. They showed that even with a full MM coupling, there are significant errors in the forces affecting atoms near the edge of the QM region

By reducing the secondary subsystem to a simple electrostatic environment, the interaction between primary and secondary subsystems is greatly simplified. In particular this scheme avoids the introduction of additional parameters necessary for MM calculations such as the Van der Waals interaction. While the dominance of the electrostatic effects is widely recognized in modeling a biomolecular environment, a direct accuracy comparison with respect to a fully QM approach is rarely found in the scientific literature. Also, partial atomic charges are typically parameterized and static for MM calculations, and the use of those for the purpose of QM embedding needs to be validated.

To address the difficulty of cutting and terminating the boundary region between the active site and the remaining protein, we evaluate the quality of various schemes to partition a protein system into a primary and secondary subsystem. We adopt the commonly used link-atom scheme, ${ }^{13}$ saturating cut dangling bonds at the QM boundary with a hydrogen atom. While there are no definitive rules about how to partition a biomolecular systems into QM and MM parts, some bonds are better candidates than other bond for cutting and capping with a link atom. Since capping atoms are usually $\mathrm{H}$ atoms (or pseudo $\mathrm{H}$ atoms) that form a $\sigma$ bond with the QM system, the most favorable bonds to cut are bonds with the similar characteristics. ${ }^{13}$ We will discuss and numerically evaluate the accuracy of cutting covalent bonds at various locations near the amide moiety in the protein backbone.

The purpose of this paper is to validate this simple electrostatic embedding approach by a quantitative evaluation of the perturbation introduced on a quantum mechanical subsystem of interest when part of it is replaced by a one-electron potential. Since our goal is in molecular dynamics simulations of the QM system, we focus on the accuracy of QM atomic forces away from the QM boundary when peptide bonds are cut and the secondary subsystem is modeled as an electrostatic potential. We study small systems such as dipeptides for which we can carry out a full reference 
QM calculation. We also pay particular attention to the problem of finding the optimal location to cut covalent bonds. This provides an assessment of the accuracy of the scheme we use to construct the one-electron potential describing the electrostatic environment, as well as a quantitative comparison between various link-atom schemes used to cut and terminate covalent bonds.

\section{Computational method}

We use density functional theory (DFT) as the QM model. While DFT has known deficiencies, in particular, to properly describe intermolecular interactions, especially van der Waals forces, it is currently one the most suitable quantum approaches for large scale electronic structure calculations in chemistry and solid-state physics (see e.g. ${ }^{14}$ ).

QM calculations were carried out using a pseudopotential finite difference approach for discretizing the Kohn-Sham equations. Electronic wave functions, potentials and the electronic density were described by their values at each grid point of a uniform real-space mesh covering the computational domain, and a fourth order finite difference scheme is used to evaluate the Laplacian in the Hamiltonian operator. ${ }^{15,16}$ Exchange and correlation were modeled using the PBE functional. ${ }^{17}$ We assumed that we have a globally neutral periodic system and use periodic boundary conditions.

One advantage of such a QM approach is the lack of basis set superposition error often present in LCAO approaches. It allows us in particular to easily assess the accuracy of our model by comparing full QM calculations with subsystems simulations. The DFT real-space finite difference approach is also very adequate for parallelism and can scale on a large number of processors, ${ }^{15}$ thus enabling calculations with a relatively large quantum region. For large systems, linear scaling can be achieved using a localized representation of the occupied Kohn-Sham orbitals subspace. ${ }^{16,18}$ Note that Takahashi et al. ${ }^{19}$ have used such an approach before for QM/MM calculations of molecules in solution.

For the molecular systems in which we are interested, we extracted a subsystem and treated 
this subsystem at the quantum mechanical level. This requires properly terminating all the covalent bonds cut during the extraction and also model appropriately the atoms outside the QM boundary. Cutting out peptide chains was done at various locations along the chain. A few possible choices were evaluated and are described in the next Section.

To model the part of the system not included in the QM simulation, we used a simple electrostatic embedding. We employed the partial charges associated to each atom in a classical force field approach. ${ }^{20}$ In several classical force fields, atoms belong to a charge group of total charge -1 , 0 or 1. To avoid overlap between QM link atoms and MM charges, we excluded charge groups that overlap with link atoms. ${ }^{21-23}$ Only charge groups that were fully cut out of the QM region and did not overlap with the termini were included in the list of charges used to generate the electrostatic potential. That usually meant excluding charges from neutral CHNH groups (backbone alpha carbon+hydrogen and amide nitrogen+hydrogen) immediately connected to a cut peptide bond. One special case was the glycine residue where the "core" charge group also includes an additional $\mathrm{H}$ atom (the side chain).

Instead of directly using the point charges associated to atoms outside the QM region, we used smeared charges. For each atom $a$ contributing to the electrostatic potential, we associated a Gaussian charge distribution

$$
\rho_{a}(r)=Z_{a} \frac{e^{-r^{2} / r_{a}^{2}}}{\pi^{3 / 2} r_{a}^{3}}
$$

where $Z_{a}$ is the classical force field partial charge of the atom, and $r_{a}$ is a radius associated to each atomic species. Values close to the covalent radii can be typically used for that purpose. We used the values listed in Table 1.

Such charge smearing has been used before, in particular for charges close to the QM boundary in order to soften the interaction between atoms that are separated by only a short distance. ${ }^{24,25}$ Indeed, at short distance, the validity of a point charge representation is questionable. In the context of this paper, as well as in a plane wave approach, ${ }^{26}$ such a smearing is a convenient way of generating a charge density and a resulting Coulomb potential representable on a uniform mesh. A total charge density associated with the atoms in the secondary subsystem was computed by 
summing up all these Gaussian charges

$$
\rho_{\text {ext }}(r)=\sum_{a} \rho_{a}(r)
$$

This total charge density was evaluated at every grid point of the real-space mesh used in the quantum calculation. The resulting Coulomb potential was calculated by solving the Poisson problem

$$
-\nabla^{2} V_{\text {ext }}(r)=4 \pi \rho_{\text {ext }}(r)
$$

with periodic boundary conditions, by discretizing the Poisson equation on the real-space mesh, the same used for electronic wave functions, by finite differences and solving the resulting linear system by the multigrid method. ${ }^{27}$ Note that even if $\rho_{\text {ext }}$ may not correspond to a neutral charge, the sum $\rho_{\text {ext }}+\rho$, where $\rho$ is the charge density of the QM part, is charge neutral. Thus, one can solve Eq.(3) for the whole system to get the total electrostatic potential without difficulties related to periodic boundary conditions by adding a uniform neutralizing background charge to $\rho_{\text {ext }}$ and subtracting it from $\rho$ in the QM calculation. $V_{\text {ext }}$ was computed only once at the beginning of the calculation and added to the Kohn-Sham potential in the QM calculation.

Since our goal is in performing molecular dynamics for the QM system, we focus in the next section on force calculations and compare forces computed in truncated systems in reference to full QM calculations. Since we did not include MM atoms, we are obviously missing important forces between atoms linked by covalent bonds at the QM frontier. To address this problem in a molecular dynamics or geometry optimization context, the coordinates of some atoms at the QM frontier should be frozen so that these erroneous forces would not be active.

Table 1: Radii used to generate Gaussian charge distributions

\begin{tabular}{cc}
\hline Species & $r_{a}[\AA]$ \\
\hline $\mathrm{H}$ & 0.40 \\
$\mathrm{C}$ & 0.73 \\
$\mathrm{~N}$ & 0.71 \\
$\mathrm{O}$ & 0.80 \\
\hline
\end{tabular}


As is the case when solving the Kohn-Sham equations with a plane wave basis, the electronic wave functions in a real-space finite difference method are in general not restricted to be local in space. As pointed out in Laio, et. al., ${ }^{26}$ carrying out such a computation in an external Coulomb potential due to MM charges can potentially lead to the so-called electron spill-out effect. While one must be aware of this issue, the calculations reported in this paper using the parameters tabulated in Table 1 showed no measurable evidence of this phenomena.

\section{Dipeptide studies}

Ferre et al. showed that it is very difficult to apply the link-atom scheme when cutting amide bonds, such as peptide bonds in a protein. ${ }^{28}$ To investigate various cutting procedures, we first quantitatively evaluate the effect of cutting and terminating a dipeptide with no net charge (Ala-Gly). To measure the effects of the Coulomb potential associated to partial charges of atoms cut out of the QM subsystem, we next study dipeptides with net charges (Arg-Asp).

\subsection{Alanine-Glycine}

We consider the dipeptide $\mathrm{H}-\mathrm{Ala}-\mathrm{Gly}-\mathrm{H}$ (Figure 1). In this form (with the H-termini), the compound is stable in gas phase and is charge neutral. We evaluate the effect of cutting the alanine out of the QM calculation. More precisely, we evaluate the atomic forces on the glycine peptide atoms after cutting out the alanine residue while properly terminating the cut peptide bond and compare these forces with those computed for the full QM system. We compare the results using the termini $\mathrm{T}=\mathrm{H}$ and $\mathrm{T}=\mathrm{NH}_{2}$. Note that a $\mathrm{NH}_{2}$ termini is equivalent to cutting the peptide chain one bond away from the peptide bond and capping the dangling bond with and $\mathrm{H}$ termini. We also look at the quantitative effect of including the Coulomb potential associated to the atoms cut out. The Coulomb potential does not include the charges of the $\mathrm{CHNH}$ group removed since some of the atoms would overlap with the termini.

Figure 2 shows the error introduced on the forces when cutting out part of the QM system 

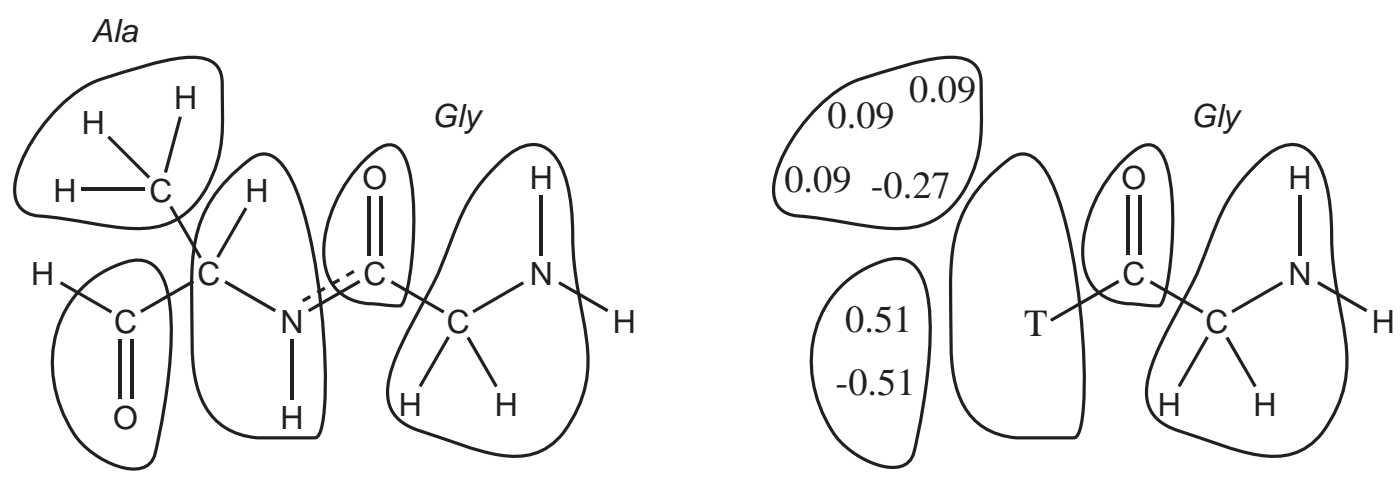

Figure 1: Left: Dipeptide Ala-Gly and the corresponding charge groups (denoted by solid contours). Right: glycine and charges substituted for alanine.

(the Ala residue). For all the atoms in the glycine part, the error is shown as a function of their distance to the (removed) alanine atoms. Numerical results show that the most important aspect of the cut is to properly terminate the cut peptide bond using an $\mathrm{NH}_{2}$ group instead of a simple $\mathrm{H}$ atom. In comparison, the effect of including the electrostatic field due to the partial charges of the removed atoms is very small. This is due to the fact that the peptide bond is not as simple as the $\mathrm{C}-\mathrm{H}$ bond. The peptide bond has two resonance forms which confers partial double character. Analyzing the electronic structure of the $\mathrm{NH}_{2}$ and $\mathrm{H}$ terminated glycine using maximally localized Wannier functions, ${ }^{29}$ one can see the different orbital character from the resulting two termini. The $\mathrm{NH}_{2}$ terminated glycine has Wannier function centers that overlap those Wannier function centers of the full system (Figure 3, left). The $\mathrm{H}$ terminated glycine, on the other hand, has a bond centered Wannier function center compared to the two off bond Wannier function centers of the full system (Figure 3, right). This confirms the observation by Ferre et al. ${ }^{28}$ that a cut peptide bond capped with a simple $\mathrm{H}$ terminus is not a suitable approach.

Another interesting result is that optimizing the geometry of the termini in the electrostatic field leads to worse geometry than optimizing in vacuo because the short distance between the termini and the Gaussian charges modeling the environment results in much too strong a coupling. Thus, optimization of termini will be done in vacuo for the test systems presented in the rest of this paper, and bond cuts will be chosen one bond away from the peptide bonds. 


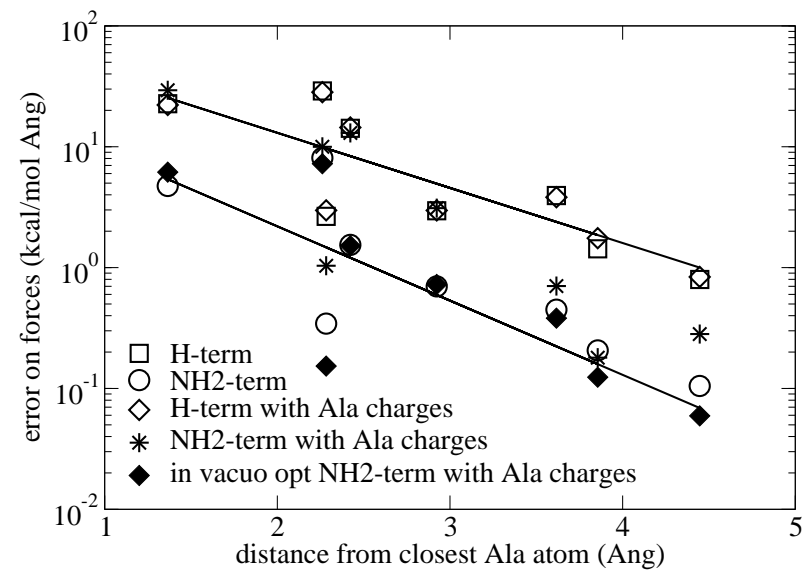

Figure 2: Absolute error on atomic forces in glycine residue when removing alanine. The lines indicate exponential fits for cases with smallest error (in vacuo opt $\mathrm{NH}_{2}$-term with Ala Charges) and largest error (H-term with Ala charges). The average errors are 9.7, 2.0, 9.7, 7.2 and 2.1 for the 5 sets of plotted data. The first data points on the left correspond to the carbon atom of alanine bonded to the termini. The next three data points correspond to the oxygen, the $\mathrm{C}_{\alpha}$ of Gly, and a hydrogen atom bonded to the $\mathrm{C}_{\alpha}$.

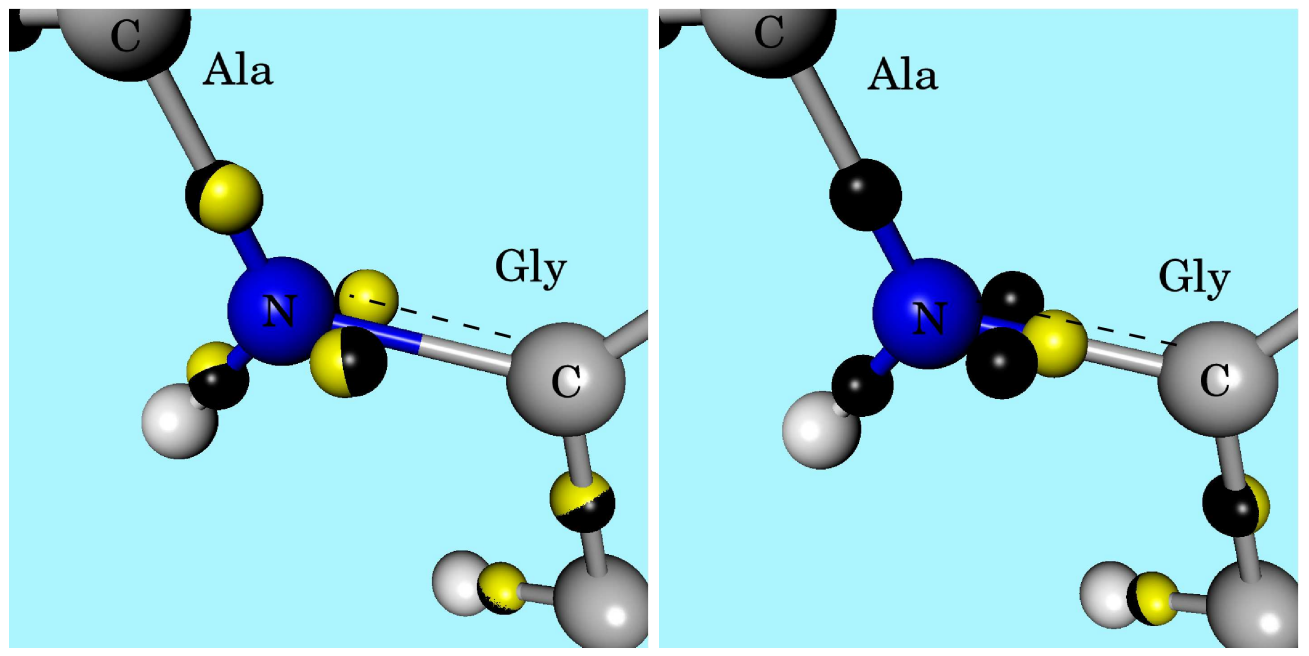

Figure 3: Centers of maximally localized Wannier functions near the peptide bond in Ala-Gly: full QM dipeptide calculation result (black balls) compared with the glycine calculation with $\mathrm{NH}_{2}$ (left) and $\mathrm{H}$ (right) termini (yellow balls). The $\mathrm{C}-\mathrm{N}-\mathrm{C}$ sequence shown correspond to the $\mathrm{C}-\mathrm{N}$ peptide bond (glycine on right) followed by the $\mathrm{C}_{\alpha}$ of alanine (removed in truncated system). 


\subsection{Arginine-Aspartate}

We now consider peptides with net electrical charges, both at their termini and in the side chain. We start with the zwitterionic Arg-Asp dipeptide (see Figure 4) solvated in water. The atomic configuration was taken from a snapshot of a classical molecular dynamics simulation. To define the reference system, we first replace the water molecules (solvent) by the Coulomb potential resulting from their partial charges according to the procedure described in Section 2. This potential is sufficiently good to have a stable quantum system made of a dipeptide with net local charges. A DFT calculation is carried out and atomic forces are computed for the whole QM system. We verify that each maximally localized Wannier center for this system closely matches the corresponding centers of the system solvated in water within $0.025 \AA$, accuracy. Next, we cut out one of the two residues and replace it with the Coulomb potential resulting from the partial charges associated to the atoms we have removed according to the procedure described in Section 2.

We began by removing Asp from the QM region (see Figure 5). The Arg residue remains intact with a net charge of +2 , while the Asp is replaced by an $\mathrm{NH}_{2}$ terminus and a Gaussian charge distribution. The $\mathrm{NH}_{2}$ terminus is equivalent to a cut one bond away from the peptide bond capped with a $\mathrm{H}$ atom. The geometry of the $\mathrm{NH}_{2}$ terminus is optimized without the electrostatic field generated by Asp charges, according to the observation made in section 3.1. Then, the Coulomb potential of the Asp charges minus the $\mathrm{CHNH}$ group, which overlaps with the terminus, is turned on (net charge -2). DFT forces were evaluated and compared with the reference calculation for the full dipeptide. Additionally, the DFT forces were compared to the result obtained when ignoring the Coulomb potential due to Asp charges (see Figure 6). The inclusion of the Coulomb potential represents a clear improvement over its deletion. The error is reduced from 5.1 to $1.6 \mathrm{kcal} / \mathrm{mol} \cdot \AA$ on average, and the trend shows an even larger improvement for atoms further away from the QM boundary. Not surprisingly, the error due to missing bonded forces dominate near the termini, while the Coulomb effect is longer range. We also conclude that the error on forces decays quickly and is within a tolerable accuracy about $3.5 \AA$ away from the removed atoms. For comparison, geometry optimization algorithms for quantum systems typically use tolerance of the order of $5 \cdot 10^{-5} a . u$. 
$(0.6 \mathrm{kcal} / \mathrm{mol} \cdot \AA)$.

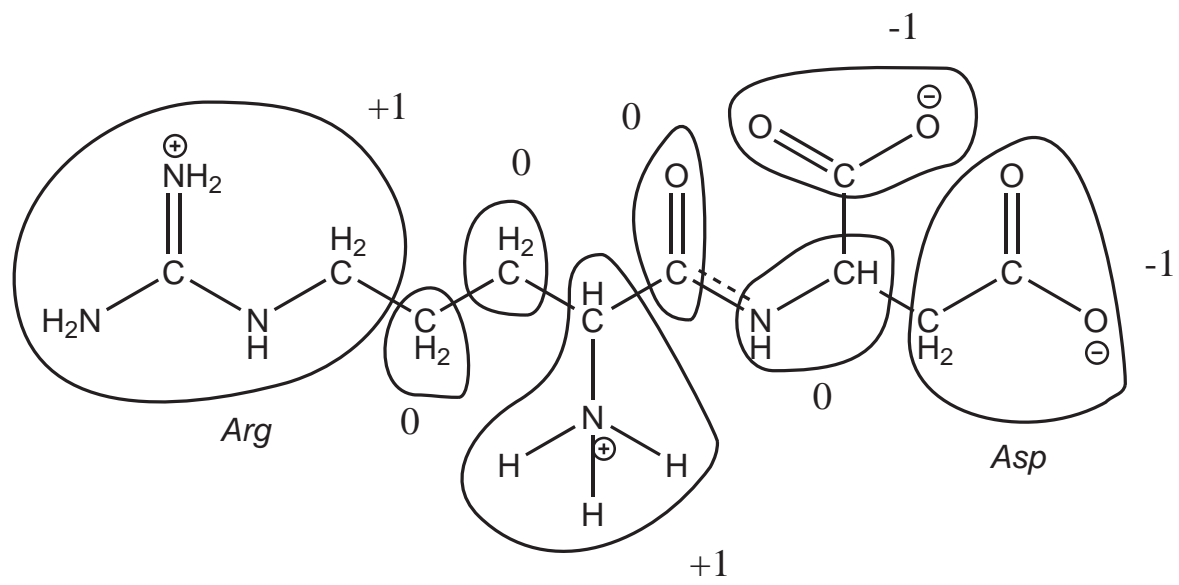

Figure 4: Dipeptide Arg-Asp and constituting charge groups.

We also carry out the same study for the case where Arg is removed and Asp is treated at the QM level. The cut is made inside Asp where the NH terminal group is replaced by a single $\mathrm{H}$ (see Figure 5). Again, we see a clear improvement when including the Coulomb potential associated to the Arg charges (Figure 6). The error is reduced from 11.9 to $6.1 \mathrm{kcal} / \mathrm{mol} \cdot \AA \AA$ on average, but the trend shows a larger improvement for atoms further away from the QM boundary. Error is reduced by a factor 10 at a distance $6.5 \AA$ from the closest Asp atom.
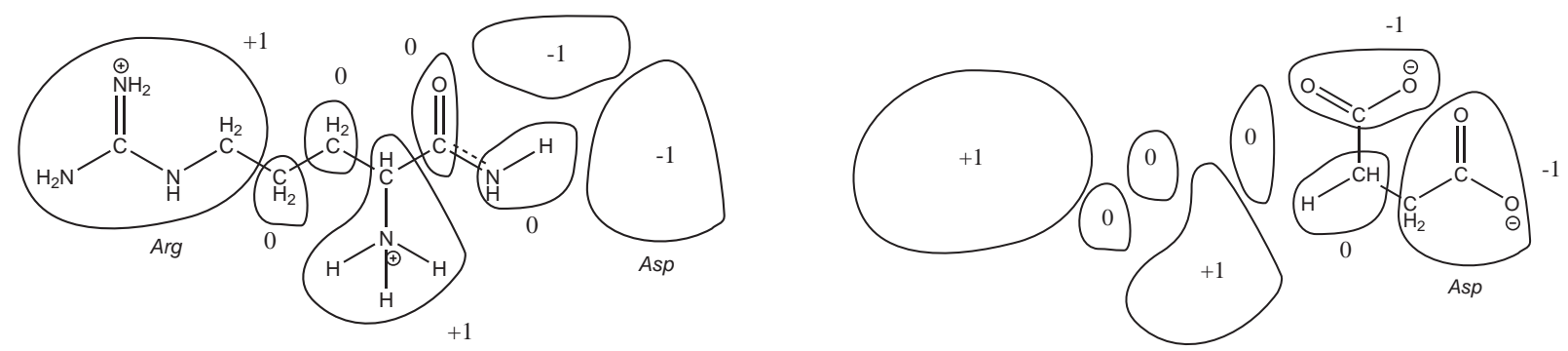

Figure 5: Truncated Arg-Asp dipeptide. Left: Arg peptide after removing Asp and replacing it with an $\mathrm{NH}_{2}$ terminus. Right: Asp peptide after removing Arg and substituting a $\mathrm{H}$ terminus for the dangling $\mathrm{NH}$ group. 

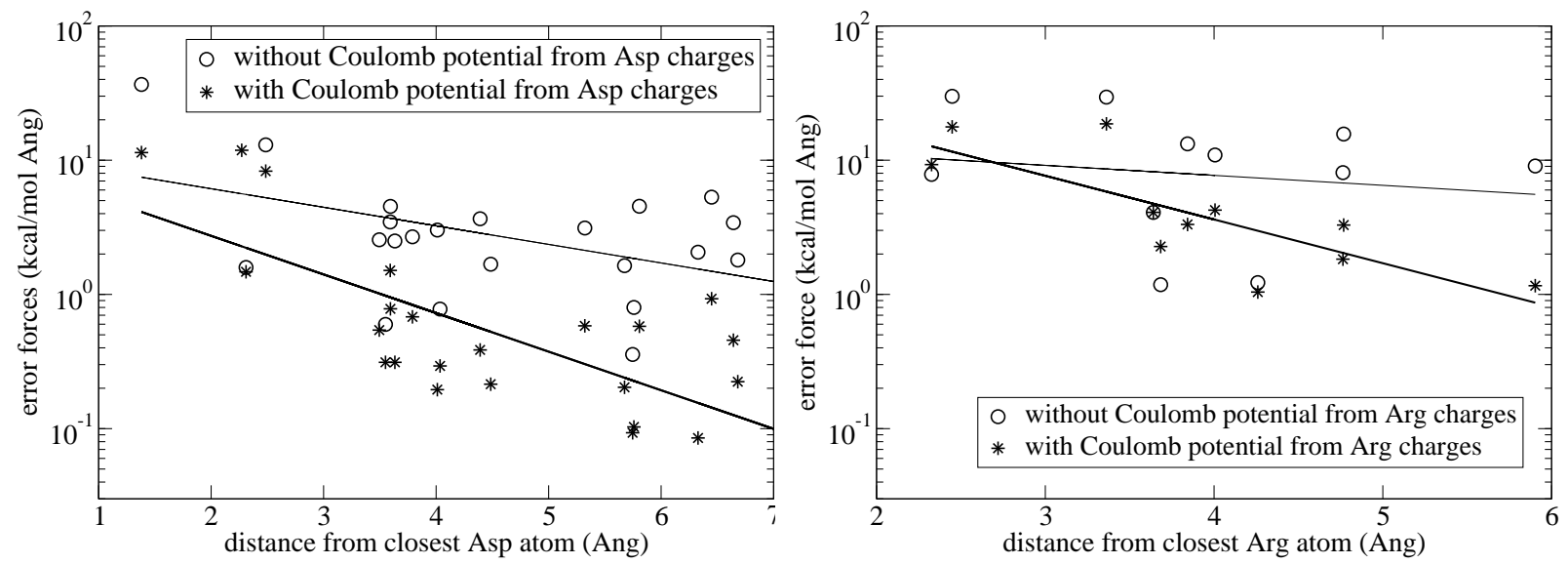

Figure 6: Absolute error on atomic forces on remaining atoms in Arg-Asp when removing Asp (Left) or Arg (right), as a function of distance from closest atom in the removed peptide. The lines indicate exponential fits for each set of results.

\subsection{Aspartate-Arginine}

We now consider the same peptide chain, but in reverse order, that is Asp on the left with a $\mathrm{NH}_{3}^{+}$ terminus and Arg on the right with a $\mathrm{COO}^{-}$terminus. In this case both residues have no net charge since their respective terminus neutralizes their charge. We have, however, on both sides a large dipole due to 2 groups with net charges compensating each other (see Figure 7). The same study as in the previous section is carried out for this system. Cut out systems are shown in Figure 8. Numerical accuracy of forces is plotted in Figure 9. While no net charge is cut out this time, the importance of including the long range Coulomb effect of the removed atoms remains. Inclusion of Coulomb charges from removed atoms reduces the error from 6.7 to $4.4 \mathrm{kcal} / \mathrm{mol}$. $\AA$ for the first system, and from 4.9 to $2.9 \mathrm{kcal} / \mathrm{mol} \cdot \AA$ for the second one. Again, we see that the error is dominated by missing bonded forces close to the QM boundary, while taking into account long range Coulomb effects allows to significantly reduce the error away from the boundary. 


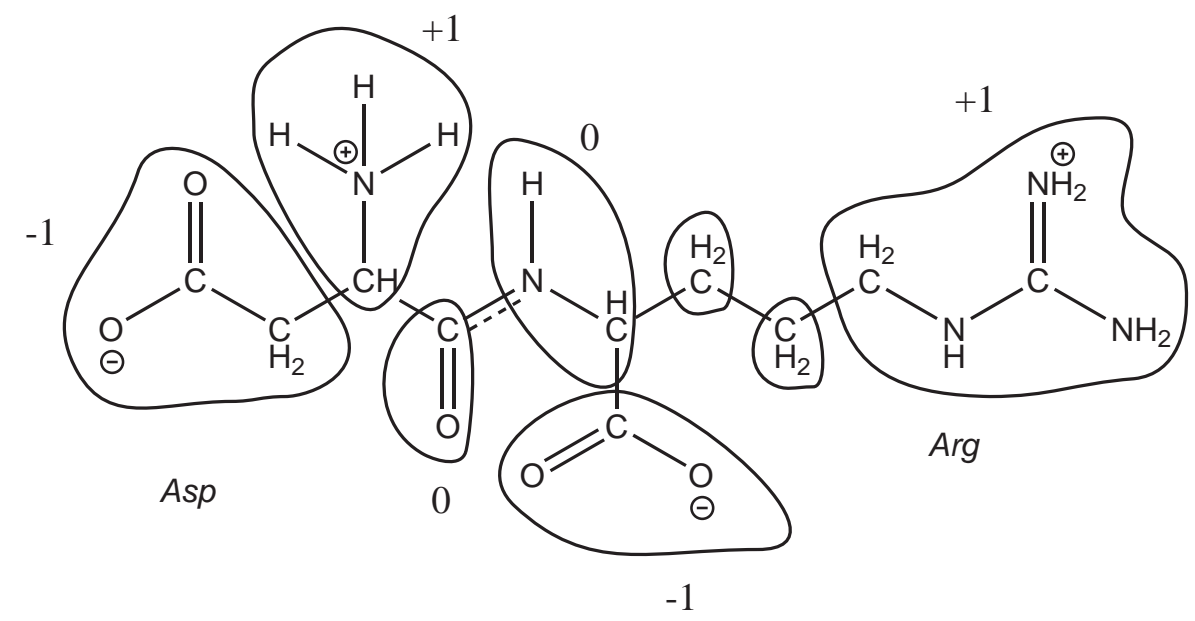

Figure 7: Dipeptide Asp-Arg and constituting charge groups.
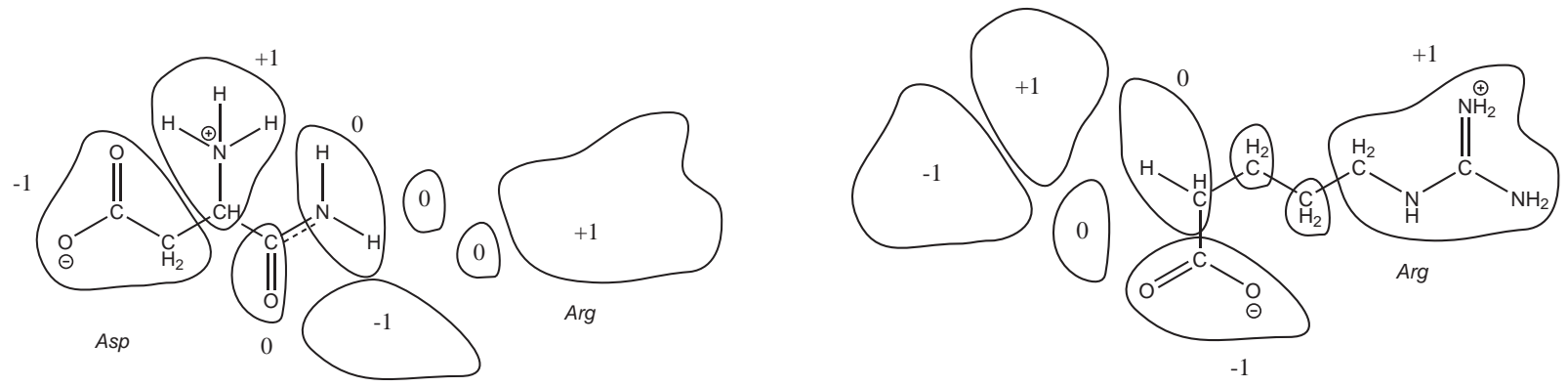

Figure 8: Truncated Asp-Arg dipeptide. Left: $\mathrm{NH}_{2}$-terminated Asp residue after removing Arg. Right: Terminated Arg peptide after removing Asp.
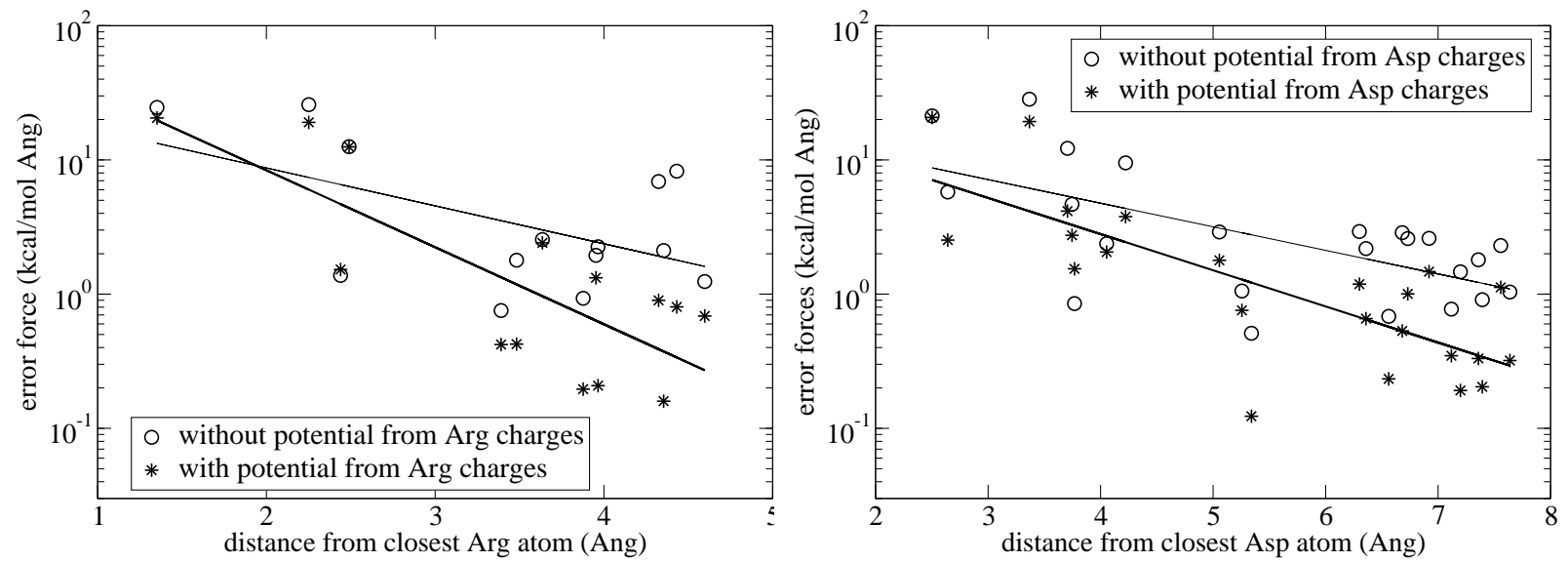

Figure 9: Absolute error on atomic forces on remaining atoms in Asp-Arg when cutting out Arg (Left) or Asp (right), as a function of distance from closest atom in cut out peptide. The lines indicate exponential fits for each set of results. 


\section{Conclusion}

In this paper we have presented an electrostatic embedding methodology for reducing QM calculations of protein systems to a small QM subsystem. The approach can be decomposed in five points: 1) cut and properly terminate peptide bonds, 2) optimize the termini atoms for the resulting QM system, 3) make a list of removed atoms, excluding those in charge groups overlapping with QM system, 4) associate each of these atoms to a Gaussian charge distribution, of charge equivalent to partial charges associated in a classical force field. 5) compute Coulomb potential associated to the sum of these charge distributions and use it as an external potential for QM calculation.

We found that the following scheme was appropriate to cut peptide bonds between the QM inside "in" region and the outside "out" region: a peptide bond "in- $\mathrm{CO}-\mathrm{NH}-$ out" is cut and terminated as "in- $\mathrm{CO}-\mathrm{NH}_{2}$ ", while the peptide bond "in- $\mathrm{NH}-\mathrm{CO}-$ out" is terminated as "in- $\mathrm{H}^{\text {". }}$ This scheme leads to much more accurate results than a simple cut of the peptide bond capped with a $\mathrm{H}$ link atom.

Our quantitative results show that the inclusion of the Coulomb potentials resulting from partial charges of atoms cut out of the primary system improves systematically and dramatically the accuracy of the calculated forces inside the QM region compared to an "in vacuo" environment. Specifically, we observe that forces on QM atoms 3.5 $\AA$ or further away from the QM boundary are quite accurate when using this procedure.

The present study shows that with a good electrostatic embedding model, QM atoms 2-3 bonds away from the QM boundary experience forces very close to those they would experience in a full QM calculation. This suggests that a simple electrostatic embedding model such as the one described in this paper is appropriate to simulate the environment for quantum biomolecular subsystems. When mechanical coupling with MM atoms is not included in the calculation, one can freeze the atomic coordinates of a "shell" of quantum atoms at the frontier of the quantum region. The resulting quantum subsystem is thus mechanically constrained at its boundary and embedded in a long range external electrostatic potential, mimicking the surrounding protein.

As mentioned in Section 2, a Generalized Gradient Approximation (GGA) was used to model 
DFT exchange and correlation in our quantum calculations (PBE functional ${ }^{17}$ ). The methodology presented in this paper for dealing with long electrostatic effects and environment modeling is however not limited to this functional and recent — and future - progress towards better functionals could be incorporated into the quantum modeling to improve the level of theory. In particular, biological systems like those considered in this paper could benefit from a better treatment of dispersion energy using for instance a Van der Waals Density Functional. ${ }^{30}$ Hybrid-GGA such as B3LYP, ${ }^{31}$ based on exact-exchange energy terms, are also quite popular in the field. While exactexchange is not as straight forward to implement in a real-space finite difference context as it is for a Gaussian basis set, recent progress in the field have shown that it is now possible to implement such functionals in a Plane Waves code. ${ }^{32}$ Even if exact-exchange makes such an approach computationally quite expensive compared to local density or generalized gradient approximations, the methodology described in Ref. ${ }^{32}$ is directly applicable to a real-space finite difference approach and should be considered as a possible future research direction.

\section{Acknowledgement}

We thank the Defense Threat Reduction Agency (BA07TAS072) for financial support. J.-L. F. would like to also acknowledge support by the Office of Science, U.S. Department of Energy, SciDAC Grant DE-FC02-06ER46262 for the development of the MGmol code used for the research presented in this paper. This work was performed under the auspices of the U.S. Department of Energy by Lawrence Livermore National Laboratory under Contract DE-AC52-07NA27344.

\section{References}

(1) Bruice, T. C. Chem. Rev. 2006, 106, 3119-3139.

(2) Mulholland, A. J. Biochemical Society Transactions 2008, 036, 22-26.

(3) Antoniou, D.; Basner, J.; Nunez, S.; Schwartz, S. D. Chem. Rev. 2006, 106, 3170-3187. 
(4) Warshel, A.; Sharma, P. K.; Kato, M.; Xiang, Y.; Liu, H.; Olsson, M. H. M. Chem. Rev. 2006, $106,3210-3235$.

(5) Siegbahn, P. E. M. Quarterly Reviews of Biophysics 2003, 36, 91-145.

(6) Himo, F. Theoretical Chemistry Accounts 2006, 116, 232-240.

(7) Henzler-Wildman, K. A.; Thai, V.; Lei, M.; Ott, M.; Wolf-Watz, M.; Fenn, T.; Pozharski, E.; Wilson, M. A.; Petsko, G. A.; Karplus, M.; Huebner, C. G.; Kern, D. Nature 2007, 450, 838-U13.

(8) Mladenovic, M.; Arnone, M.; Fink, R.; Engels, B. J. Phys. Chem. B 2009, 113, 5072-5082.

(9) Lin, H.; Truhlar, D. G. Theoretical Chemistry Accounts: Theory, Computation, and Modeling (Theoretica Chimica Acta) 2007, 117, 185-199.

(10) Senn, H.; Thiel, W. QM/MM Methods for Biological Systems. In Atomistic Approaches in Modern Biology; Springer: Berlin/Heidelberg, 2007; Vol. 268, pp 173-290.

(11) Elstner, M. Theor. Chem. Acc. 2006, 116, 316-325.

(12) Solt, I.; Kulhánek, P.; Simon, I.; Winfield, S.; Payne, M. C.; Csányi, G.; Fuxreiter, M. The Journal of Physical Chemistry B 2009, 113, 5728-5735.

(13) Field, M. J.; Bash, P. A.; Karplus, M. Journal of Computational Chemistry 1990, 11, 700733.

(14) Hafner, J.; Wolverton, C.; Ceder, G. MRS bulletin 2006, 31, 659-668.

(15) Briggs, E. L.; Sullivan, D. J.; Bernholc, J. Phys. Rev. B 1996, 54, 14362-14375.

(16) Fattebert, J.-L.; Bernholc, J. Phys. Rev. B 2000, 62, 1713-1722.

(17) Perdew, J. P.; Burke, K.; Ernzerhof, M. Phys. Rev. Lett. 1996, 77, 3865-3868.

(18) Fattebert, J.-L.; Gygi, F. Phys. Rev. B 2006, 73, 115124. 
(19) Takahashi, H.; Hori, T.; Hashimoto, H.; Nitta, T. J. Comp. Chem. 2001, 22, 1252-1261.

(20) Jr., A. D. M.; Brooks, B.; III, C. L. B.; Nilsson, L.; Roux, B.; Won, Y.; Karplus, M. CHARMM: The Energy Function and Its Parameterization with an Overview of the Program. In The Encyclopedia of Computational Chemistry; John Wiley \& Sons: Chichester, 1998; Vol. 1, pp 271-277.

(21) Eurenius, K. P.; Chatfield, D. C.; Brooks, B. R.; Hodoscek, M. Int. J. Quant. Chem. 1996, 60, 1189.

(22) Antes, I.; Thiel, W. On the treatment of link atoms in hybrid methods. In Combined Quantum Mechanical and Molecular Mechanical Methods; American Chemical Society Publication, 1998; pp 50-65.

(23) Lyne, P.; Hodoscek, M.; Karplus, M. Journal of Physical Chemistry A 1999, 103, 3462-3471.

(24) Logunov, I.; Schulten, K. Quantum chemistry of in situ retinal: Study of the spectral properties and dark adaptation of bacteriorhodopsin. In Proceedings of the Ecole de Physique des Houches; Bicout, D., Field, M. J., Eds.; Springer: Paris, 1995; pp 235-256.

(25) Amara, P.; Field, M. J. Theoretical Chemistry Accounts 2003, 109, 43-52.

(26) Laio, A.; VandeVondele, J.; Rothlisberger, U. J. Chem. Phys. 2002, 116, 6941.

(27) Briggs, W. L.; Henson, V. E.; McCormick, S. F. A multigrid tutorial (2nd ed.); Society for Industrial and Applied Mathematics: Philadelphia, PA, USA, 2000.

(28) Ferre, N.; Olivucci, M. Journal of Molecular Structure: THEOCHEM 2003, 632, 71-82.

(29) Marzari, N.; Vanderbilt, D. Phys. Rev. B 1997, 56, 12847.

(30) Thonhauser, T.; Cooper, V. R.; Li, S.; Puzder, A.; Hyldgaard, P.; Langreth, D. C. Phys. Rev. B 2007, 76, 125112. 
(31) Stephens, P. J.; Devlin, F. J.; Chabalowski, C. F.; Frisch, M. J. J. Phys. Chem. 1994, 98, 11623-11627.

(32) Wu, X.; Selloni, A.; Car, R. Phys. Rev. B 2009, 79, 085102. 